\title{
An Analytical Approach for the Selection of Technologies to Be Integrated in a Plug\&play Façade Unit: The RenoZEB Case Study ${ }^{+}$
}

\author{
Alessandro Pracucci 1,*, Sara Magnani 1, Laura Vandi 1, Oscar Casadei 1, Amaia Uriarte ${ }^{\text {, }}$ \\ Bruno Bueno ${ }^{3}$ and Michele Vavallo ${ }^{4}$ \\ 1 Focchi S.p.A., Via Cornacchiara, 47824 Poggio Torriana Rimini, Italy; s.magnani@focchi.it (S.M.); \\ l.vandi@focchi.it (L.V.); o.casadei@focchi.it (O.C.) \\ 2 Tecnalia Research \& Innovation, Parque Tecnológico de Bizkaia, Astondo Bidea, Edificio 700, 48160 Derio, \\ Spain; amaia.uriarte@tecnalia.com \\ 3 Fraunhofer Institute for Solar Energy Systems ISE, Heidenhofstr. 2, 79110 Freiburg, Germany; \\ bruno.bueno.unzeta@ise.fraunhofer.de \\ 4 R\&D Department, Solintel M\&P S. L. Avda. de la Industria n. 4, edificio 1, Oficina 1B, 28108 Alcobendas, \\ Madrid, Spain; michele.vavallo@solintel.eu \\ * Correspondence: a.pracucci@focchi.it \\ † Presented at the Sustainable Places 2020, Online, 28-30 October 2020; Available online: \\ https://www.sustainableplaces.eu/. \\ Published: 18 January 2021
}

\begin{abstract}
The nearly Zero Energy building (nZEB) renovation market is currently the key feature in the construction sector. RenoZEB aims to develop a systematic approach for retrofitting by assembling different technologies in a plug and play building envelope. This paper presents the methodology used to transform the RenoZEB concept in the design system. A multi-criteria decision matrix is used for the selection of the best façade technologies within the market while the analysis of the existing building conditions allows to develop a replicable approach for designing deep retrofitting intervention through a plug\&play façade. The methodology appears to be a valuable support for the selection of technologies and allows to define a design guideline for the envelope.
\end{abstract}

Keywords: energy-efficient buildings; methodology; plug\&play façade; multi-criteria decision matrix

\section{Introduction}

The RenoZEB project [1] is a proposal to the call Horizon2020 [2] started in 2017 which involved 19 European partners. The aim of the RenoZEB project is to establish a systematic methodology for the deep retrofitting of energy for building stock markets by developing a BIM-based platform and tools for actors of the value chain. The project is referred to as the nearly Zero Energy Building (nZEB) renovation market by increasing property value through a new systemic approach for retrofitting. RenoZEB includes innovative components, processes, and decision-making methodologies to guide all value-chain actors in the nZEB building renovation by developing a cost-effective plug and play solution for a large-scale deep nZEB retrofitting scheme. The entire process is controlled by a digital platform which guarantees the replicability and the adaptability of the retrofitting intervention. In order to enable the methodology, a plug\&play façade is composed by different macro technologies. The aim of this paper is to explain the method adopted to identify the best and suitable technologies for RenoZEB Plug\&Play (P\&P) façade solution by using an analytic approach for concept design and by analyzing the existing building conditions. The definition is to develop a prefabricated façade, 
manufactured off-site with the purpose of reduce the time, the cost, and to improve the final quality of renovation. The method, described in the article, has been used to move from the RenoZEB P\&P concept to the engineering design to achieve project purposes.

\section{Methods}

The RenoZEB P\&P façade integrates multiple on-market technologies and the first analysis conducted focused on their selection to identify the best solution in line with the RenoZEB P\&P façade concept. The analytic approach developed to design the RenoZEB P\&P façade system is composed by the following steps:

1. Technologies identification-The identification of the best façade technologies found within the market and ranked into a multi-criteria decision matrix (MCDM) (Figure 1) regarding the RenoZEB P\&P façade technological system and its components.

2. Boundary conditions - The analysis of the existing building boundary condition was conducted to define a baseline for the RenoZEB P\&P façade application in building and consequently addressing its design.

1. REQUIREMENTS

2.TECHNOLOGIES

\begin{tabular}{|c|c|c|c|c|}
\hline \multirow[b]{2}{*}{ Requirements } & \multicolumn{4}{|c|}{ Proposed component/façade } \\
\hline & 1 & 2 & 3 & 4 \\
\hline \multicolumn{5}{|l|}{ Ventilated façade } \\
\hline \multicolumn{5}{|l|}{ Plug and play } \\
\hline \multicolumn{5}{|l|}{ Low-cost multifunctional insulation boards } \\
\hline \multicolumn{5}{|l|}{ Commercially available PV modules } \\
\hline \multicolumn{5}{|l|}{ Commercially available solar thermal collectors } \\
\hline \multicolumn{5}{|l|}{ "Click in system" for air ducts, heat exchangers } \\
\hline \multicolumn{5}{|l|}{ Integration of hydraulic, electric and HVAC installations } \\
\hline \multicolumn{5}{|l|}{ Modular, Pre-cast and Easy Assembly-Disassembly } \\
\hline minimize on-site work & & & & \\
\hline improve product quality & & & & \\
\hline reduce costs & & & & \\
\hline \multicolumn{5}{|l|}{ Industrialization } \\
\hline minimize on-site work & & & & \\
\hline cost reduction & & & & \\
\hline improve quality & & & & \\
\hline increased safety & & & & \\
\hline \multicolumn{5}{|l|}{ Less intrusive system } \\
\hline prio ritize outdoor interventions & & & & \\
\hline avoid interruption in the dwellings & & & & \\
\hline reduce the duration of the interventions & & & & \\
\hline off-site manufacturing & & & & \\
\hline plug-and-play solutions & & & & \\
\hline optimized building processes & & & & \\
\hline \multicolumn{5}{|l|}{ Aesthetic and functional integration } \\
\hline harmonizing architectonic rehabilitation with social acceptance & & & & \\
\hline expectations of the architects & & & & \\
\hline $\begin{array}{l}\text { Integration and adaptation of multifunctional } \\
\text { insulation system }\end{array}$ & & 3 & SCORE SY & STEM \\
\hline use of recycled material & & & & \\
\hline recyclability of the final solution & & & & \\
\hline reduction of heat and pressure losses & & & & \\
\hline fire protection & & & & \\
\hline prefabricated window insulation frames & & & & \\
\hline minimum of heat losses & & & & \\
\hline \multicolumn{5}{|l|}{ Integration of thermal and PV modules } \\
\hline integration into the RenoZEB envelop and building concept & & & & \\
\hline \multicolumn{5}{|l|}{ Connection elements } \\
\hline Integrate subsystems in a holistic approach & & & & \\
\hline \multicolumn{5}{|l|}{ Development of the Smart-IoT façade module } \\
\hline Low-cost sensors & & & & \\
\hline Low-intrusive installation & & & & \\
\hline Embedding in the module & & & & \\
\hline Integration with PV and battery system & & & & \\
\hline Data collections system with common protocols & & & & \\
\hline Plug \& play solution & & & & \\
\hline Points achieved & & & & \\
\hline
\end{tabular}

Figure 1. Multi-criteria decision matrix (MCDM). 


\subsection{Technologies Identification}

\subsubsection{Multi-Criteria Decision Matrix (MCDM)}

The MCDM is set on the requirements and outputs expected within the RenoZEB concept and it has been used to direct the choices related to the RenoZEB P\&P façade, with the purpose to standardize the final solution towards the strategies the project aims at enhancing. The matrix is composed by 4 sections; the first column reports the main requirements and eventually subrequirements in italic, in the columns there are the market competitors for each category and the relative evaluations. The fourth part is related to the last line with mathematical sums to the given scores. The evaluation corresponds to each RenoZEB solution and project requirement. The score system is described in the following table (Table 1).

Table 1. Evaluation system used in the multi-criteria decision analysis (MCDA).

\begin{tabular}{ccc}
\hline \multicolumn{3}{c}{ Correspondence } \\
\hline Degree & Value & Symbol \\
\hline Not Applicable (N/A) & 0 & 0 \\
Low & 1 & $\bullet$ \\
Medium & 2 & $\bullet \bullet$ \\
High & 3 & $\bullet \bullet \bullet$ \\
\hline
\end{tabular}

\subsubsection{Multi-Criteria Decision Analysis (MCDA)}

Once the technologies are ranked, the matrix is analyzed in an analytic way to identify the component with the highest evaluation. This is a qualitative method which gives an overall score based on project requirements to each current market components. The selected components could be integrated in the RenoZEB P\&P façade solution. Within the RenoZEB P\&P façade, the existing building represents, with its structural and technological elements, the reference for the analysis and for this reason only façade systems and technologies which do not require the removal of existing building façade were evaluated. Different technological solutions for building retrofitting have been considered (double-skin façade, curtain wall with stick system and unitized systems [3]).

In line with the RenoZEB P\&P façade concept, the technologies analyzed are:

- Prefabricated window module and roller shutter;

- Multifunctional insulation board (Figure 2);

- Ventilation devices;

- Building Integrated Photovoltaics (BIPV) and batteries;

- Building Integrated Solar Thermal (BIST);

- Hybrid solution for BIPV and BIST.

The matrix in is an example for the MCDA conducted for each technology: 


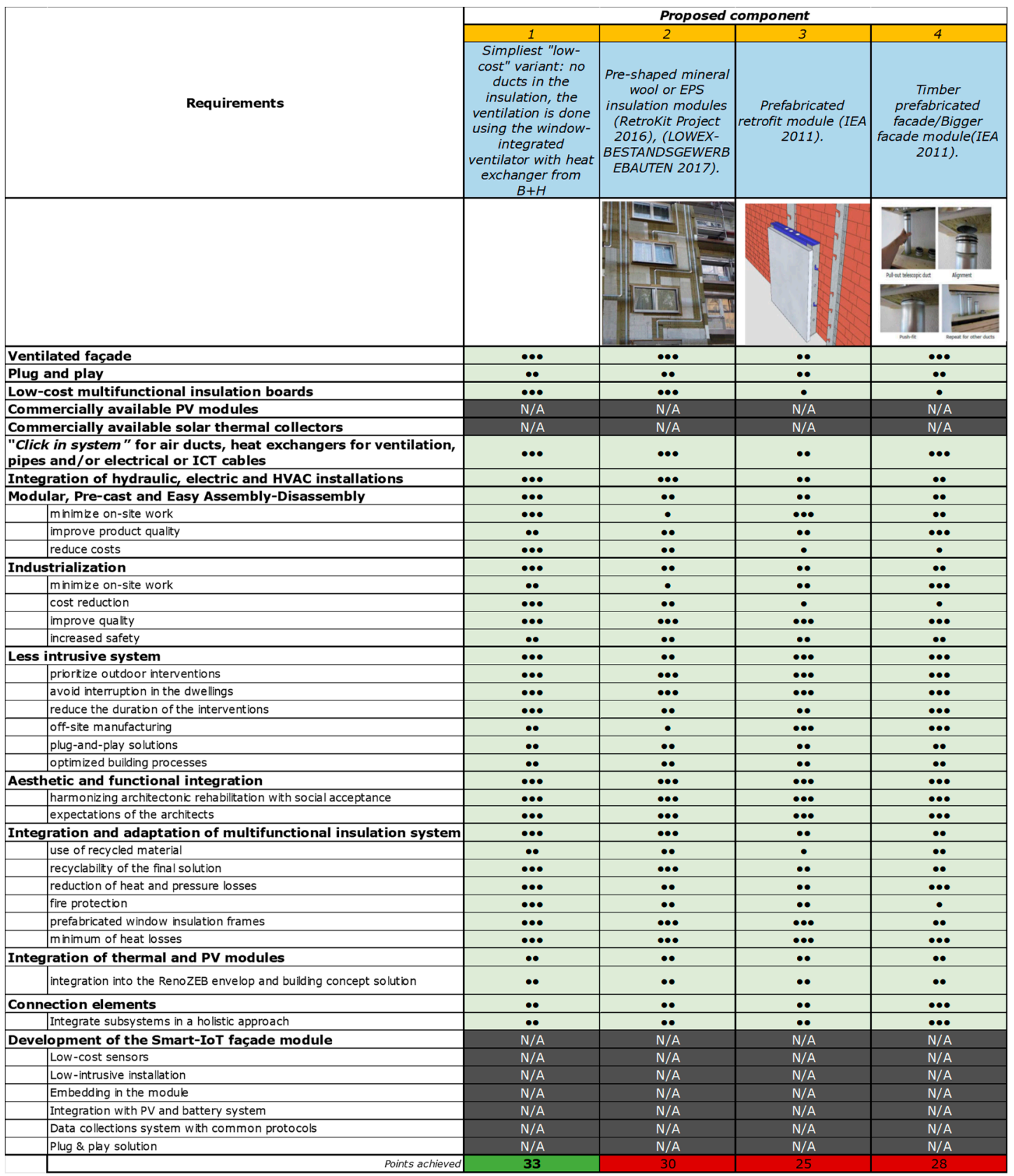

Figure 2. MCDM for multifunctional insulation board.

\subsection{Boundary Conditions}

The second step of the methodology is to define the building boundary conditions as the building typology and structural system to develop a replicable approach for designing deep retrofitting intervention through the prefabricated RenoZEB P\&P facade. Working on existing buildings through refurbishment activities in a less intrusive manner without affecting inner users' daily activities (RenoZEB expected outputs) directs to consider those elements-technological, structural-that cannot be changed, representing fixed boundary conditions for the new envelope design. In this frame, window openings, structural elements (slabs, cavity walls), load capacity of structural elements, and technological characteristics of the envelope (material specifications, width, etc.) can be included. By analyzing the boundary conditions, it could be possible to identify the baselines to design the additional RenoZEB P\&P façade units. Based on boundary conditions, the concept design uses these elements as guidelines to design the module that will constitute the RenoZEB envelope system. This baseline will be the parameter to be included as reference in the BIM model. 


\section{Results}

This section resumes and concretizes the main aspects of the concept design. Despite MCDA offering an analytic analysis of components that could be installed and applied in RenoZEB envelope system, it is important to read these results considering the full view of the concept. It is central to cross the results of the MCDA of the envelope system with the MCDA for the components, to define the most valuable solutions for the project itself. The utilization of the MCDA as a standalone system could cause mismatches between solutions with problems in further concept design phases.

Considering the above for the main aspects of the RenoZEB concept, the role of industrialization and off-site manufacturing of the envelope system is central, and the prevailing analysis is the one of the technological systems of the envelope.

The results are listed in the following table (Table 2):

Table 2. Selection of the most suitable technologies.

\begin{tabular}{ll}
\hline \multicolumn{1}{c}{ Technological Component } & RenoZEB Envelope System's Component \\
\hline $\begin{array}{l}\text { Prefabricated window module and roller } \\
\text { shutter }\end{array}$ & Compact SA \\
Multifunctional insulation board & Telescopic ducts \\
Ventilation devices & Centralized ventilation system with air \\
Building Integrated Photovoltaics (BIPV) & ducts in insulation layer \\
and batteries & BIPV \\
Building Integrated Solar Thermal & BIST with air \\
Façade controller & FENER webtool \\
Façade-integrated sensors & Solar radiation and light sensors \\
Fixing mechanisms & TBD during system design on specific \\
& building structure \\
\hline
\end{tabular}

Once the technological system and the components to be embedded in the RenoZEB envelope system are defined, the method defines key elements for the designing process of the façade module and the further façade system design. Six different phases were used in the adopted method (Figure 3):

1. Existing building-Identification of the building object of renovation.

2. Boundary conditions-This phase identifies the elements that are typical of the building of reference: building typology and structural system.

3. Definition of baseline-The baseline is defined by the opening's dimensions and on the amount and location of fixing mechanisms with building structure.

4. Primary modules design-The first component to keep in consideration are the windows, thus the first primary modules are designed depending on the elements which do not change position during the renovation intervention.

5. Secondary modules design - The secondary modules are designing based on the primary one, and these modules could include the following components: PV components, solar thermal components, HVAC system's ducts.

6. Modules aggregation - It is a crucial phase because it provides the reduction of installation time and on-site activities. The modules are joined and designed to guarantee the requested performances of the envelope system as well as the best transport optimization.

In addition, regarding the module design, a multifunctional insulation system depends on the expected result of the refurbishment. The solutions could be only insulation, ventilation devices, air ducts, wire for electrical system, PV battery and wire for electrical system, as well as thermal collector fluid ducts. The module is closed with a cladding system which could be a technical system (PV and solar thermal collector), or an external finishing material selected among different ones based on the cost, material appraisal, and local sources. 


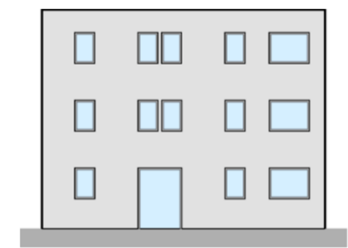

Phase 1-existing building

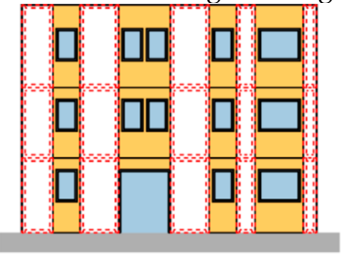

Phase 4-primary modules designed

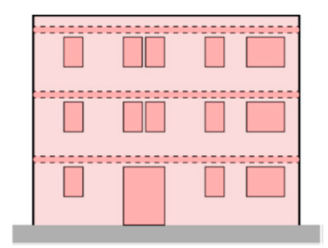

Phase 2-boundary conditions

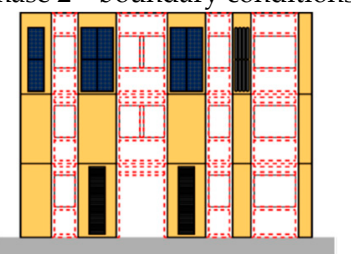

Phase 5-secondary modules designed

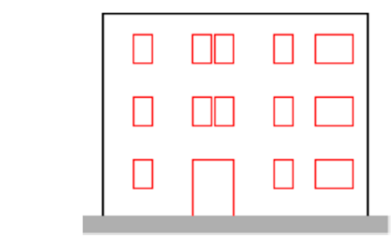

Phase 3-identification of baseline

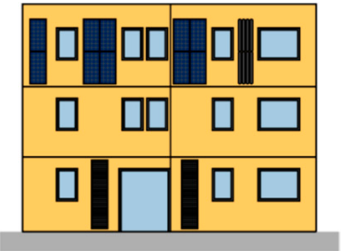

Phase 6-module aggregation design

Figure 3. Phases for the design system.

\section{Conclusions}

The analytics approach presented appears to be valuable for a qualitative definition of market products to be integrated in a façade plug\&play solution as the one defined in RenoZEB. Indeed, the market and customer requirements can be well-defined with this method and their correspondence with products' advantages, moving from a concept to design phase with a preliminary assessment of technologies in line with the final product outcome. In addition, the method defines a guideline for the design process for the Plug \& Play façade. Despite this, the approach could be further implemented for quantitative analysis to better support the further engineering phase. Therefore, the qualitative approach is a limited characteristic of the methodology.

Author Contributions: Conceptualization, A.P.; methodology, A.P.; resources, M.V., A.U., A.P., B.B., L.V., O.C., and S.M.; data curation, M.V., A.U., A.P., B.B., L.V., O.C., and S.M.; writing-original draft preparation, L.V. and A.P.; writing-review and editing, A.P., L.V., A.U., B.B., and M.V.; visualization, A.P. and L.V.; project administration, M.V. and A.P.; funding acquisition, M.V. All authors have read and agreed to the published version of the manuscript.

Funding: This research has received funding from the European Union's Horizon 2020 research and innovation program under Grant Agreement No. 768718.

Acknowledgments: The authors want to thank the RenoZEB partners for the fruitful discussions and collaboration.

Conflicts of Interest: The authors declare no conflict of interest.

\section{References}

1. RenoZEB in a Nutshell-RenoZEB. Available online: https://renozeb.eu/about/renozeb-in-a-nutshell.html (accessed on 16 November 2020).

2. Horizon 2020. Available online: https://ec.europa.eu/programmes/horizon2020/en (accessed on 16 November 2020).

3. Focchi Group-Curtain Walls. Available online: https://www.focchi.it/ww/ (accessed on 11 January 2021).

Publisher's Note: MDPI stays neutral about jurisdictional claims in published maps and institutional affiliations.

(C) 2021 by the authors. Licensee MDPI, Basel, Switzerland. This article is an open access article distributed under the terms and conditions of the Creative Commons Attribution (CC BY) license (http://creativecommons.org/licenses/by/4.0/). 